\title{
Editorial
}

\section{Advanced Functional Nanomaterials Synthesis and Nanocrystal Growth Technology}

\author{
Wei-Chun Chen, ${ }^{1}$ Hao-Chung Kuo, ${ }^{2}$ Rajanish Nath Tiwari, ${ }^{3}$ Shou-Yi Kuo, ${ }^{4}$ \\ Fang-I Lai, ${ }^{5}$ and Ying-Lung Daniel $\mathrm{Ho}^{6}$ \\ ${ }^{1}$ Instrument Technology Research Center, National Applied Research Laboratories, Hsinchu City, Taiwan \\ ${ }^{2}$ Department of Photonics, National Chiao Tung University, Hsinchu City 30076, Taiwan \\ ${ }^{3}$ Department of Electrical and Electronic Engineering, Faculty of Science and Technology, Saga University, Saga 840-8502, Japan \\ ${ }^{4}$ Department of Electronic Engineering, Chang Gung University, Taoyuan City 33302, Taiwan \\ ${ }^{5}$ Department of Photonics Engineering, Yuan Ze University, Taoyuan City 32003, Taiwan \\ ${ }^{6}$ Department of Electrical and Electronic Engineering, University of Bristol, Bristol BS8 1TH, UK
}

Correspondence should be addressed to Wei-Chun Chen; weichun@narlabs.org.tw

Received 18 May 2015; Accepted 20 May 2015

Copyright (C) 2015 Wei-Chun Chen et al. This is an open access article distributed under the Creative Commons Attribution License, which permits unrestricted use, distribution, and reproduction in any medium, provided the original work is properly cited.

Functional materials at the nanoscale have received increasing attention in their demand, application, and development in recent years because of their dominant role in our science and society.

Also advanced functional nanomaterials synthesis and crystal growth technology are exciting systems for a wide variety of applications. Due to their advantageous properties, their use has increased over the recent years in industrial and environmental applications and their impact can be significant. The environmental fate of nanoparticles is often complex however requiring detailed knowledge of their stability, chemistry, migration, accumulation, and toxicity, whilst delivering superior performance in their intended functional capacity. A wide range of researchers from civil engineering, water science, geology, environmental science, chemistry, materials science, and ecotoxicology are involved in this emerging multidisciplinary field. With their unique structures and novel properties, they cover a wide range of technologies, such as solid state lighting based on lightemitting diodes (LED), green energy electronics based on solar cell devices, nanocomposites, and biomedical engineering. Therefore, the topic, for researchers and graduate students, combines the novelty of nanomaterials synthesis and crystal growth technology and generality of basic ideas relating to nanoscience and nanotechnology. Considering movement towards future nanomaterials synthesis, we have collected papers which cover the topical areas of new activities in advanced functional nanomaterials synthesis and crystal growth technology and published these in this special issue on advanced functional nanomaterials synthesis and crystal growth technology.

This special issue provides some new research and developments in nanomaterials. It presents the principles of the synthesis and fabrication of nanostructures and their applications and also some important tools and challenges associated with these techniques for engineers and scientists.

This special issue contains 8 research articles, including papers on synthesis of inorganic nanocomposites and analysis and synthesis of cuprous oxide quantum dots, sulfonyldiamine-Ru complex, single-layer graphene, and thin films growth. For synthesis of nanomaterials, the papers are explaining some novel approaches to enhance the optical and physical parameters of material with different techniques. For instance, one paper describes a facile suspension and solid phase method for the synthesis of surfactants-free $\mathrm{Cu} 2 \mathrm{O}$ QDs at high precursor quantity.

For thin films growth, the papers are about the thin films and their physical properties. One tried to describe how ultrathin ITO films can be used as the touch sensors. In addition, for the $\mathrm{Cu}$ interconnection process of current integrated 
circuit (IC) devices, TaN barrier shows that the diffusion barrier fabricated by the combination of crystallized $\alpha-\mathrm{Ta}$ and TaN with high $\mathrm{N}$ concentration efficiently reduces the $\mathrm{KRc}$ and improves the EM resistance of $\mathrm{Cu}$ interconnection structure.

We hope this special issue with a small collection of papers can provide the readers with an overview of the recent progress achieved and the future developing directions in the nanomaterials synthesis and crystal growth technology. We sincerely hope that this special issue could provide a valuable reference and perspective for the research community working in this exciting field and inspire many more to enter it.

Wei-Chun Chen

Hao-Chung Kuo

Rajanish Nath Tiwari

Shou-Yi Kuo

Fang-I Lai

Ying-Lung Daniel Ho 

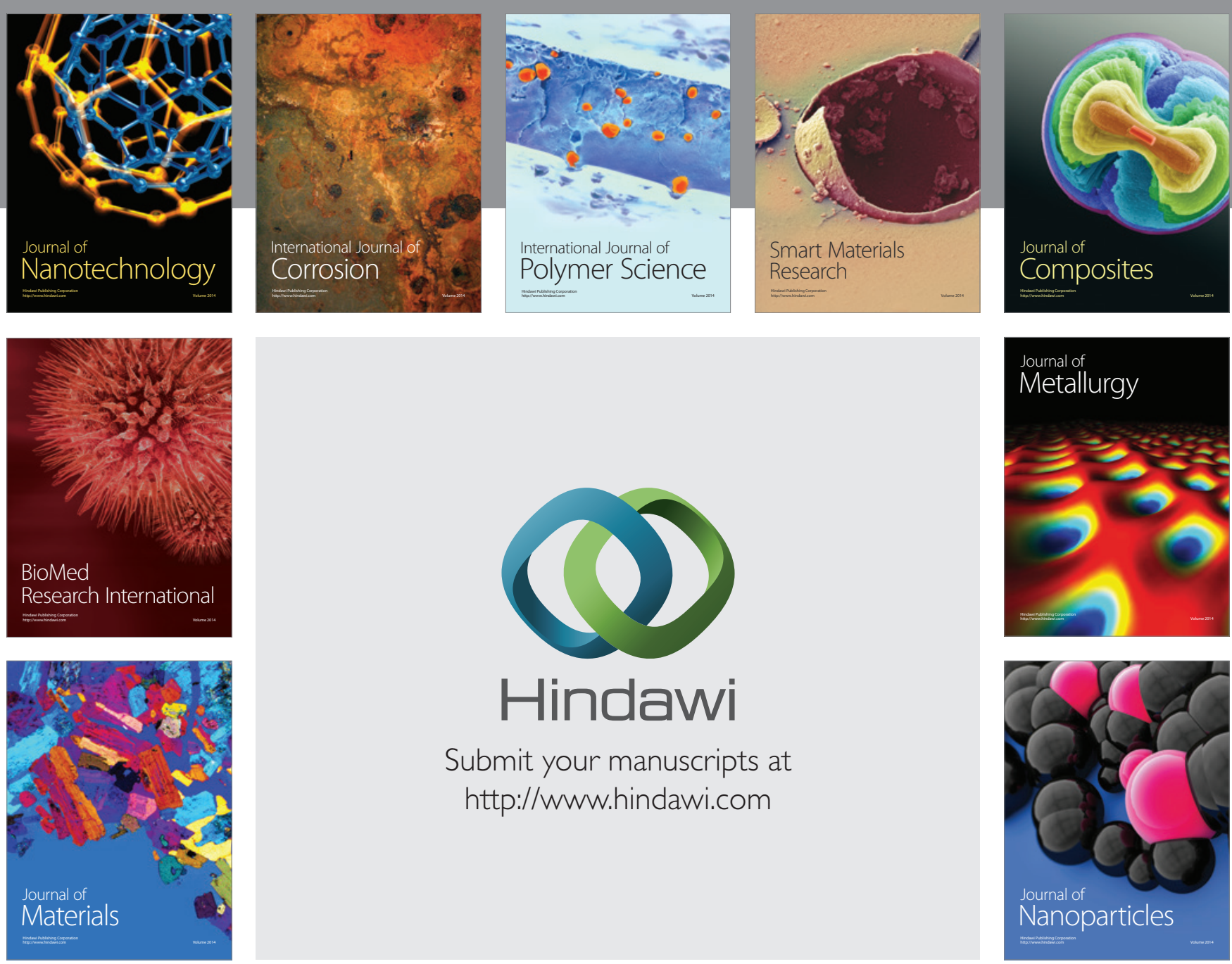

Submit your manuscripts at http://www.hindawi.com
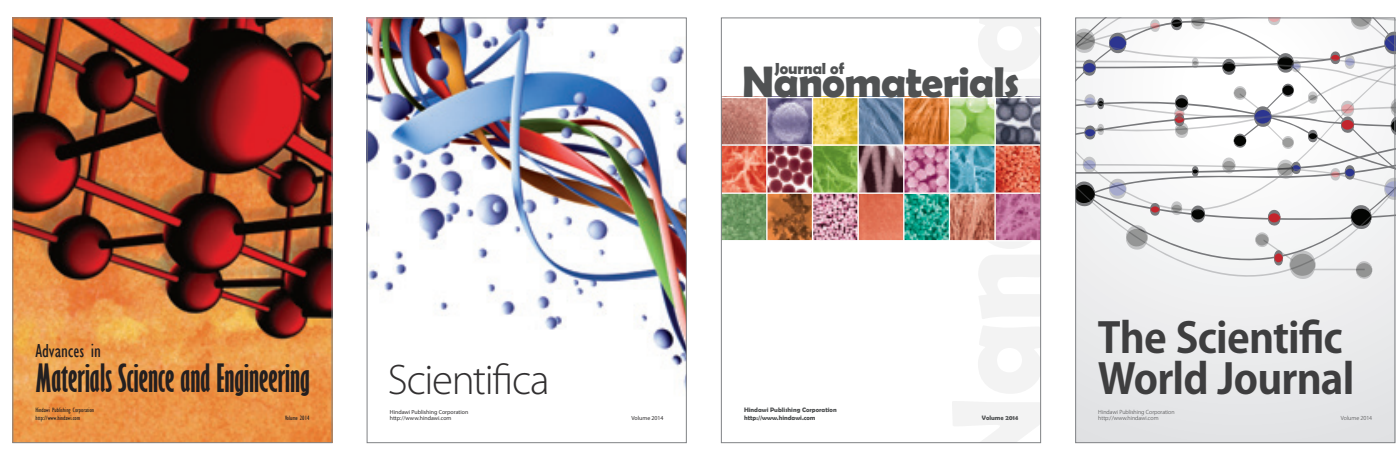

\section{The Scientific World Journal}
